\title{
Desarrollo de bioplásticos a partir de tortas residuales y gomas naturales
}

\author{
Javier Sayavedra-Delgado, Roman Rodríguez-Maecker \\ Departamento de Energía y Mecánica, Universidad de las Fuerzas Armadas ESPE, Sangolquí, Ecuador \\ jjsayavedra@espe.edu.ec, rnrodriguez@espe.edu.ec.
}

\begin{abstract}
Resumen-El objetivo de este estudio fue la generación de biopolímeros extraídos de tortas residuales para la elaboración de películas biodegradables. La harina que se obtiene de estas tortas desengrasadas puede usarse como una fuente viable y de bajo costo en la formación de películas biodegradables. Se usaron a su vez, gomas naturales que favorezcan y mejoren las propiedades de las películas tanto físicas como mecánicas. Las películas resultantes fueron evaluadas en propiedades físicas (permeabilidad al vapor de agua, espesor), se evaluó el efecto de la concentración de las gomas guar, tragacanto y xantana en dos niveles 0,05 y $0,08 \mathrm{~g}$, en las propiedades de barrera que ofrecen las películas para este estudio se enfocó en la permeabilidad al vapor de agua. Se utilizó películas sin ningún tipo de goma como películas de control para ver el efecto de las gomas en las propiedades. La solubilidad de las películas se encontró entre $5,32 \%$ y $33 \%$, el contenido de humedad entre 9 y $18 \%$.
\end{abstract}

Palabras Claves-Biopolímeros, biodegradación, tortas residuales.

Abstract-The objective of this study was the generation of biopolymers extracted from residual cakes for the production of films. The flour obtained from these degreased cakes can be used as a viable and inexpensive source in the formation of biodegradable films. It is used in its place, natural gums that favor and improve the properties of both physical and mechanical films. The resulting films were evaluated in physical properties (water vapor permeability, thickness), the effect of the concentration of guar, tragacanth and xanthan gums at two levels 0.05 and $0.08 \mathrm{~g}$, on the barrier properties was evaluated the films offered for this study focused on water vapor permeability. Films without any gum have been used as control films for the effect of gums on the properties. The solubility of the films was between $5.32 \%$ and $33 \%$, the moisture content between 9 and $18 \%$.

Keywords—Biopolymers, biodegradation, residual cake

\section{INTRODUCCIÓN}

A partir del surgimiento del plástico y de su industrialización, un gran porcentaje de los mismos se transformaron en residuos contaminantes. Los polímeros provenientes de los derivados del crudo de petróleo se han vuelto indispensables en el diario vivir, como materiales comunes de envasado, debido a sus características como ligereza, resistencia, transparencia y maleabilidad. En la actualidad, la comodidad característica de la sociedad ha generado un consumo excesivo de productos desechables, elaborados de plásticos, por lo que año tras año se generan alrededor de 500 billones y un trillón de bolsas plásticas, de las cuales apenas el 1\% es reciclado. El 75,20\% de hogares ecuatorianos utilizan fundas plásticas desechables para realizar sus compras diarias y el 22,38\% son hogares que toman consciencia y utilizan bolsas ecológicas que son hechas de material reutilizable o tela.[1]
Se pretende buscar una alternativa para aplicaciones específicas de vida corta como envasado de alimentos.[2] Varios de los procesos que se han desarrollado a la fecha en base a la Química Verde están presentes aproximadamente en todas las áreas de la química, como por ejemplo la orgánica, inorgánica, bioquímica, polimérica, ambiental, toxicológica, física, etc. La química verde no es una disciplina científica separada, sino que realza la responsabilidad interdisciplinaria de las ciencias, basándose en la responsabilidad química, ecológica y social. [3]

La torta que es un subproducto de la industria del aceite posee un alto contenido proteico. Las proteínas son biomoléculas poco solubles o insolubles en agua, formadas por cadenas lineales de aminoácidos que se pliegan adquiriendo una estructura tridimensional que amplía la gama de propiedades funcionales, el más importante es la unión a nivel intermolecular. Las proteínas son distintas y dependiendo su origen pueden variar en peso molecular, arreglo espacial, cargas eléctricas, flexibilidades y estabilidad térmica. [4]

Los biopolímeros procedentes de recursos renovables es una nueva alternativa a la utilización de empaques.[5] La síntesis de nuevos materiales poliméricos a partir de recursos renovables naturales se ha convertido en un área de rápido crecimiento, ya que estos materiales podrían reemplazar total o parcialmente los plásticos desfavorables para el medio ambiente y la energía derivados de productos químicos del petróleo.[6] Sin embargo, las aplicaciones de polímeros renovables están significativamente detrás de los polímeros derivados del petróleo, en parte debido al costo relativamente alto y limitaciones en los recursos de monómeros, por lo tanto, polímeros derivados.

Las gomas naturales son gomas provenientes de plantas, químicamente son polímeros hidrofílicos de carbohidratos de alto gran peso molecular. Tienen la capacidad de absorber agua he hincharse, debido a que pueden solubilizarse y dispersarse en agua, hasta obtener una solución viscosa. [7]

Los plásticos biodegradables son aquellos en los cuales la degradación es el resultado de la acción de macroorganismos naturales como bacterias, hongos y algas. el compostaje es un proceso administrado que controla la descomposición biológica y la trasformación de materiales biodegradables que luego dará como resultado la producción de dióxido de carbono, agua, minerales y humus o compost.[8]

Este proyecto busca desarrollar películas de origen orgánico empleando biopolímeros obtenidos de las tortas residuales de las semillas oleaginosas que son un subproducto de la extracción de aceites vegetales. También se pueden añadir gomas naturales como reforzamiento de las 
películas, lo que mejora sus propiedades físicas y mecánicas como fuerza de tensión, permeabilidad, etc.

\section{MÉtodo}

Se empleó tortas residuales de la extracción de aceite de semillas empleadas industrialmente, las mismas que fueron secadas, trituradas, tamizadas y desengradas. Se realizó un análisis de contenido de proteína mediante el método AOAC 991.20.[9] La proteína fue calculada multiplicando el contenido de nitrógeno por el factor de conversión 6,25.[9][10]

\section{A. Extracción de la proteína de las tortas residuales}

Se realizó la extracción de proteína de la torta desengrasada por método álcali. Se mezcló las tortas de ajonjolí, soya y sacha inchi en agua destilada en vasos de precipitación, en razón de 1:10, 1:15 y 1:10, respectivamente. Luego con la ayuda de un $\mathrm{pH}$ metro (Modelo BT 7, BOECO, Alemania) se ajustó el pH de las soluciones a 11, 9 y 12 para ajonjolí, soya y sacha inchi, usando una solución $2 \mathrm{M}$ de $\mathrm{NaOH}$ y se agitó continuamente mediante un agitador magnético con placa calefactora (Modelo MSH 420, BOECO, Alemania) donde se deja solubilizar la proteína por alrededor de una hora y posteriormente se centrifugó a 4000 rpm durante 2 minutos. Se filtró el sobrenadante y se ajustó hasta el punto isoeléctrico $\mathrm{pH}$ 4,5 para las tres tortas con la ayuda de una solución $2 \mathrm{M}$ de HCl. Luego se centrifugó a 4000 rpm por 15 minutos. El precipitado se suspendió en agua destilada y se ajustó a pH 7 y se almacenó a $4{ }^{\circ} \mathrm{C}$ durante una noche. Transcurrida la noche la solución se descartó y se secó al vacío.[11]

\section{B. Preparación de las películas.}

El método de formación de películas empleado es el de “casting” o moldeo, donde en la colada o solución se disuelve o dispersa el polímero, se recubre sobre un sustrato de soporte, y luego el agua o el disolvente se elimina por secado para crear una capa sólida sobre el soporte. La capa de colada resultante se puede separar del sustrato de soporte para producir una película independiente.[12]

Para la preparación se utilizó la proteína extraída de cada torta. Se trabaja con $9 \%(\mathrm{p} / \mathrm{v})$ en relación a $100 \mathrm{~mL}$ de solución formadora. La cantidad utilizada de glicerol como plastificante es de $10 \%$ con respecto a la cantidad de proteína. El pH de formación fue de 12 para ajonjolí, sacha inchi y 10 para soya. La solución se calentó a baño maría hasta $90^{\circ} \mathrm{C}$. la solución filmogénica se filtró y se colocó en moldes para su secado a $35^{\circ} \mathrm{C}$.[13]

Para las películas con goma natural se realiza el mismo procedimiento, con la adición de 0,05 y 0,08g de las gomas en la colada.

\section{ANÁLISIS DE RESULTADOS}

La concentración óptima de la formación de películas se estableció mediante el análisis de la permeabilidad al vapor de agua, debido que es una propiedad más crítica pues en los envases de alimento, se debe buscar la disminución de la transferencia de humedad entre la atmosfera y el alimento [14].

En la Fig. 1, se puede apreciar que la combinación que posee menor permeabilidad al vapor de agua es la AG005 (Proteína de ajonjolí con 0,05 g de goma guar), la interacción de la goma guar con la proteína de ajonjolí permite que el arreglo intermolecular actué de mejor manera contra el vapor de agua. Por el contrario, la goma tragacanto favorece al paso del vapor de agua. Con referente a la película de control este valor de AG005, es bajo por lo que se infiere que la goma favorece la disminución de la permeabilidad del vapor de agua. Los resultados establecen las relaciones entre los datos obtenidos, el problema de investigación, el método y el soporte teórico de la revisión de la literatura.

\section{Películas biodegradables}

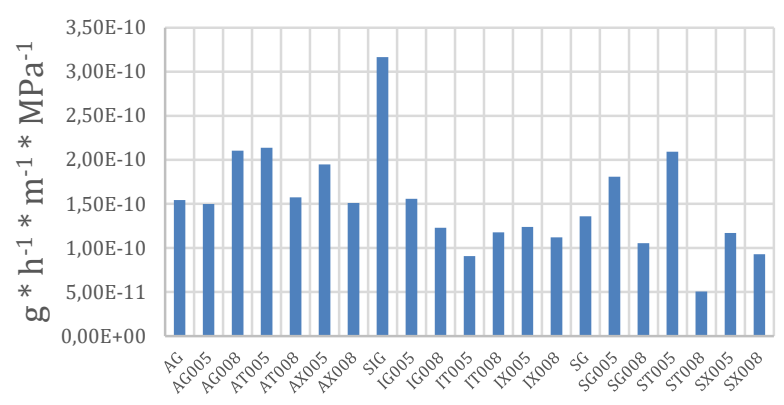

Fig. 1. Grafica de permeabilidad al vapor de agua.

\section{A. Ensayo mecánico}

El ensayo de tracción se desarrolló por triplicado, asimismo se realizó solo de la película que presentó mejor propiedad de barrera en este caso se evaluó la permeabilidad al vapor de agua.

En la Fig. 2, se puede divisar el comportamiento de la película en función de la fuerza aplicada, esta curva se divide en 3 secciones que son: sección 1, corresponde a las deformaciones elásticas como su nombre lo dice elásticas es decir, que si se retira la carga aplicada la probeta regresaría a su forma inicial, además es la sección donde rige el módulo de elasticidad o de Young; la sección 2 es de cedencia, donde se produce una deformación brusca de la probeta manteniéndose la carga aplicada, esto se produce por debido a impurezas presentes en la probeta que obstaculizan el deslizamiento característico de la deformación elástica; sección 3 deformación plástica, donde sí se retira la carga aplicada la probeta no recupera su forma original sino parcial evidencia cierta deformación.[15]

El valor obtenido de módulo de elasticidad fue de 11,418, el esfuerzo máximo alcanzado de 7,74 $\mathrm{MPa}$ y el porcentaje de elongación antes de la rotura fue de 50\%. 


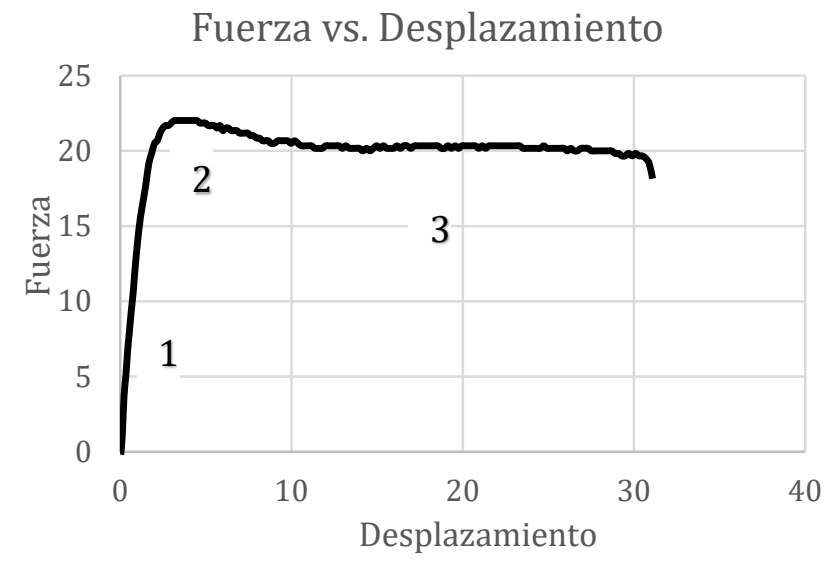

Fig. 2. Grafica de resistencia a la tracción

\section{B. Análisis termogravimétrico}

En la Fig. 3, se muestran curvas de comparación entre la película proteicas de soya con su película de control (sin goma natural). Como se especificó en la metodología antes de realizar todos los ensayos las películas fueron colocadas en una atmosfera de 55-60\% de humedad relativa durante 48 horas. Dicho esto, se justifica la perdida inicial de todas las películas se atribuye a la perdida de humedad y deshidratación hasta $130{ }^{\circ} \mathrm{C}$.

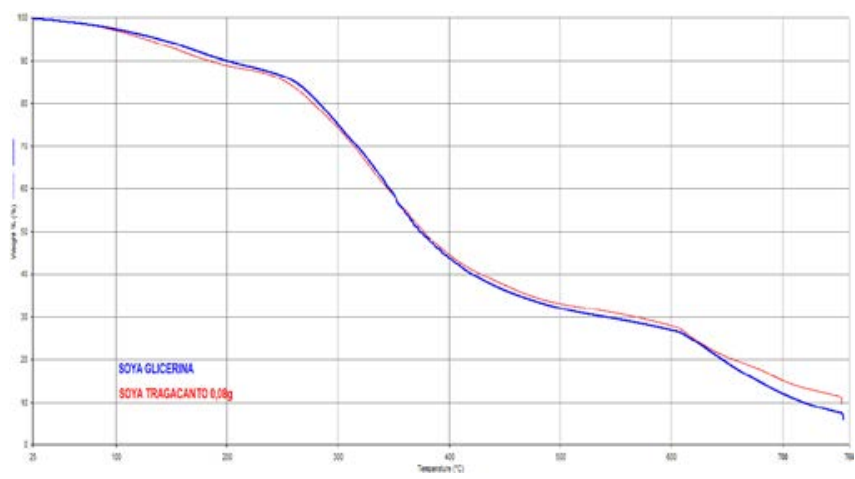

Fig. 3. Termograma de la película soya - tragacanto.

La Tabla I presenta una comparación del comportamiento de las películas estableciendo rangos de temperatura definidos por literatura, donde se infiere los procesos ocurridos en el ensayo para películas biodegradables.

TABLA I

RESUMEN DEL ANÁLISIS TERMOGRAVIMÉTRICO DE PELÍCULAS ÓPTIMAS

\begin{tabular}{|c|c|c|c|}
\hline$\Delta \mathbf{T}\left({ }^{\circ} \mathbf{C}\right)$ & \multicolumn{2}{|c|}{ \% Peso perdido } & \multirow[t]{2}{*}{ Proceso } \\
\hline & ST008 & SG & \\
\hline $25-170$ & 7,3 & 8,676 & $\begin{array}{l}\text { Perdida de humedad y } \\
\text { deshidratación. }\end{array}$ \\
\hline $220-340$ & 26,507 & 26,268 & $\begin{array}{l}\text { Perdida de plastificante y aditivos } \\
\text { (gomas naturales). }\end{array}$ \\
\hline $340-600$ & 33,406 & 35,417 & $\begin{array}{l}\text { Descomposición de polisacáridos } \\
\text { y aminoácidos presentes en la } \\
\text { proteína. }\end{array}$ \\
\hline $610-750$ & 15,415 & 18,539 & $\begin{array}{l}\text { Incineración de todo el material } \\
\text { combustible. }\end{array}$ \\
\hline \% Residuo & 9,714 & 6,095 & Contenido de cenizas \\
\hline \multicolumn{4}{|c|}{ ST008: Proteína de soya con 0,08 g de goma tragacanto } \\
\hline \multicolumn{4}{|c|}{ SG: Proteína de soya con glicerina(control) } \\
\hline
\end{tabular}

Este análisis realizó con el fin de obtener información sobre la estructura de las películas y las interacciones entre las proteínas de ajonjolí, sacha inchi y soya y las gomas guar, tragacanto y xantana. El análisis FTIR se realizó para la materia prima, es decir, las proteínas, junto con las películas de control y las películas que presentan menor permeabilidad al vapor de agua.

Las posiciones de los picos de absorción característicos primarios facilitan la comparación de los picos de absorción de los bioplásticos de control y los bioplásticos compuestos. Esto se utilizó como confirmación de los cambios estructurales de la proteína extraída antes y después del procesamiento, así como para resaltar las interacciones entre las proteínas y gomas naturales. La curva de absorción infrarroja de la proteína de sacha inchi y soya contienen picos de absorción de vibración de estiramiento N-H y O-H que ocurren a 3271 y $3280 \mathrm{~cm}^{-1}$, respectivamente.[13]

Mientras que las vibraciones de estiramiento $\mathrm{C}-\mathrm{H}$ de los grupos CH3 y CH2 ocurrieron a $2932 \mathrm{~cm}-1$ para ambas proteínas. A 1634 cm-1 de proteína de sacha inchi y 1635 cm-1 en proteína de soya, se observaron picos de absorción de la flexión de $\mathrm{H}-\mathrm{N}-\mathrm{H}$, y vibraciones de estiramiento $\mathrm{C}=\mathrm{O}$ en las bandas amida I', mientras que a 1526, 1236 para la proteína de sacha inchi y 1523 y 1236 para la proteína de soya, se distinguen los picos de flexión y absorción de $\mathrm{NH}$ en bandas de amida II y vibraciones de estiramiento $\mathrm{CN}$ en la banda de amida III. A 1072 y $1067 \mathrm{~cm}-1$, se produjo el pico de absorción de vibraciones del enlace glicosídico C-OC que es parte de la estructura de la proteína.[16]

Al realizar la comparación de la materia prima, bioplástico de control y la bioplástico junto con la goma se observó variaciones en 2 posiciones como se aprecia en la Fig. 4, el pico de absorción $2932 \mathrm{~cm}^{-1}$, ocurre un aumento a $2959 \mathrm{~cm}^{-1}$ esto puede deberse al cambio de estructura molecular por la desnaturalización de la proteína por efecto del calentamiento. El segundo cambio se da en la variación de 1072 a $1106 \mathrm{~cm}^{-1}$, que es atribuible a la disociación de las proteínas, con el cambio de estructura del enlace C-C de la estructura de anillo y del enlace glicosídico C-O.

\section{CONCLUSIONES}

Se desarrolló bioplásticos a base de biopolímeros obtenidos de tortas residuales de la industria de aceite y gomas naturales mediante el método de casting o moldeo, que resultaron maleables, sin olor, el color se asocia a la fuente de proteína, las condiciones de formación de bioplástico se escogieron de estudios realizados a partir de proteína donde se obtuvieron las mejores condiciones para la formación de bioplásticos como $\mathrm{pH}$, temperatura y agitación. El bioplástico más viable no cambia su estructura de manera notable con respecto al espectro de la proteína y de la bioplástico de control. Estos resultados demostraron que las proteínas extraídas de la torta de Soya y Sacha Inchi tienen gran potencial como material formador de bioplásticos biodegradables/comestibles con propiedades adecuadas para su uso como envases y/o embalaje.

\section{AGRADECIMIENTOS}

Un agradecimiento al Laboratorio de Petroquímica de la Universidad da las Fuerzas Armadas-ESPE, por su 


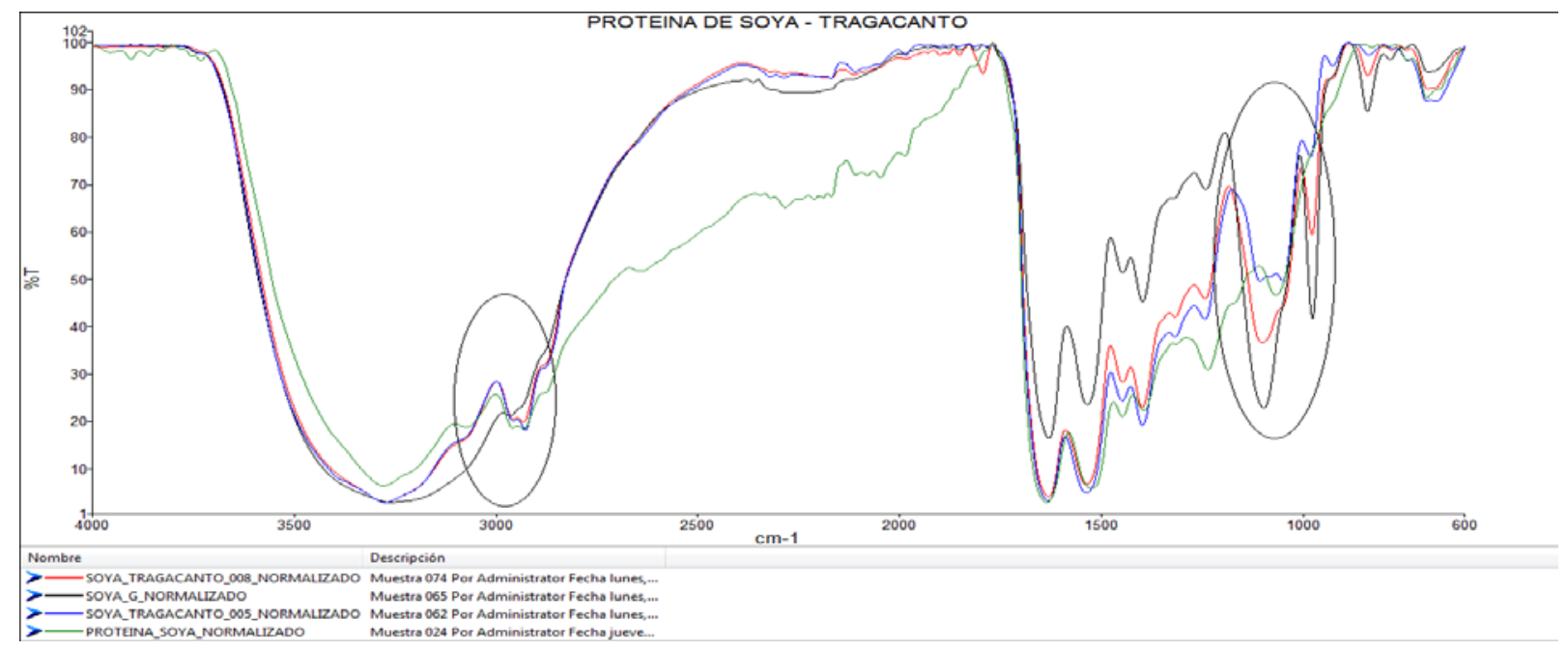

Fig. 4. Espectro FTIR de la bioplástico de proteína de soya con goma tragacanto en el intervalo de 4000-600 cm-1.

colaboración que fue de vital importancia para el desarrollo de la presente investigación.

\section{REFERENCIAS}

[1] N. Sánchez, "Estadisticas sobre las bolsas plásticas,” 2010. [Online]. Available: https://www.veoverde.com/2010/03/estadisticas-sobre-lasbolsas-plasticas/.

[2] T. Janjarasskul and J. M. Krochta, "Edible Packaging Materials," Annu. Rev. Food Sci. Technol., vol. 1, no. 1, pp. 415-448, 2010.

[3] A. Ivanković, A. Dronjić, A. M. Bevanda, and S. Talić, "Review of 12 Principles of Green Chemistry in Practice Review of 12 Principles of Green Chemistry in Practice,” no. July, 2017.

[4] N. Gontard, C. Duchez, J. Cuq, and S. Guilbert, "Edible Composite Films of Wheat Gluten and Lipids - Water-Vapor Permeability and Other Physical-Properties,” Int. J. Food Sci. Technol., vol. 29, no. 1 , pp. 39-50, 1994.

[5] B. Saberi, S. Chockchaisawasdee, J. B. Golding, C. J. Scarlett, and C. E. Stathopoulos, "Physical and mechanical properties of a new edible film made of pea starch and guar gum as affected by glycols, sugars and polyols,” Int. J. Biol. Macromol., vol. 104, pp. 345-359, 2017.

[6] J. D. Badia, O. Gil-Castell, and A. Ribes-Greus, "Long-term properties and end-of-life of polymers from renewable resources," Polym. Degrad. Stab., vol. 137, pp. 35-57, 2017.

[7] S. Goswami and S. Naik, "Natural gums and its pharmaceutical application,” J. Sci. Innov. Res. JSIR, vol. 3, no. 31, pp. 112-121, 2014.

[8] ASTM D6400, "Standart Specification for Compostable Plastics," in American Society for Testing and Materials, vol. i, 2004, pp. 4-6.

[9] J. M. Lynch and D. M. Barbano, "Kjeldahl nitrogen analysis as a reference method for protein determination in dairy products.," J. AOAC Int., vol. 82, no. 6, pp. 1389-98, 1999

[10] “AOAC Official Method 998.06,” 2002.

[11] L. Sharma and C. Singh, "Composite Film Developed From Blends of Sesame Protein Isolate and Gum Rosin and Their Properties Thereof," Polym. Polym. Compos., vol. 16, no. 2, pp. 101-113, 2016.

[12] S. Sadhukhan, "Preparation and Characterization of Polymer Electrolyte,” 2011.

[13] C. Sui, W. Zhang, F. Ye, X. Liu, and G. Yu, "Preparation, physical, and mechanical properties of soy protein isolate/guar gum composite films prepared by solution casting,” J. Appl. Polym. Sci., vol. 133, no. 18, pp. 1-9, 2016.

[14] N. T. Nemet, V. M. Šošo, and V. L. Lazić, "Effect of glycerol content and $\mathrm{pH}$ value of film-forming solution on the functional properties of protein-based edible films,” Acta Period. Technol., vol. 41, pp. 5767, 2010.

[15] V. M. Azevedo et al., "Effect of replacement of corn starch by whey protein isolate in biodegradable film blends obtained by extrusion," Carbohydr. Polym., vol. 157, pp. 971-980, 2017.

[16] J. Piermaria, A. Bosch, A. Pinotti, O. Yantorno, M. A. Garcia, and A. G. Abraham, "Kefiran films plasticized with sugars and polyols: water vapor barrier and mechanical properties in relation to their microstructure analyzed by ATR/FT-IR spectroscopy,” Food Hydrocoll., vol. 25, no. 5, pp. 1261-1269, 2011. 